\title{
Interception of Class-III malocclusions
}

\author{
Y. Soyer \\ Qualified Specialist in DFO, former Assistant Hospital-University, Hospital Practitioner, \\ Hôpital Pitie-Salpêtrière Paris, Expert for the Court of Appeal of Paris
}

\section{SUMMARY}

Class-III malocclusions are characterized by an excessively anterior position of the mandible in relation to the maxilla and present a wide variety of clinical forms accompanied by esthetic prejudice.

The purpose of interceptive therapy is to restore normal oro-facial functions to limit the need for orthognathic surgery.

The early implementation of treatment is the key to success.

\section{KEYWORDS}

Class-III malocclusion, early treatment, orthopedic treatment, missed opportunity

\section{INTRODUCTORY REMARKS}

When I met Mrs. Henriette Muller for the first time in May 1983, she quickly confided to me her interest in the tongue's role in Class-III anomalies.

But we could easily get distracted discussing the spirit and vivacity of this great lady of orthodontics.

Retrospectively, before the Revue d'ODF (DFO Journal) published several articles on Class-III malocclusions in 1970, Henriette Muller (Madame Louis Muller) found that there was a scarce amount of literature on the subject. This disenchantment was, for her, the fruit of failures on the part of the orthodontists considering this anomaly was the unfortunate source of many inevitable relapses. The issue of this review published in January 1970, which would be followed in April and July of the same year by several articles on therapeutic possibilities as well as the surgical treatments of Class-III malocclusions, laid the groundwork for a dialog that Henriette Muller would continue in the third issue with Jean Delaire dealing with mandibular prognathism ${ }^{6}$.

The Revue d'ODF would then regularly publish articles on the subject of interception and early treatment of Class-III malocclusions, and a whole issue would be fully devoted to it in 2003. Educated in "Functional Orthodontics" by André Salvadori ${ }^{24}$, it was under his tutelage that we published articles on interception in 1987 for the 20th Anniversary of the Revue ${ }^{25}$.

Address for correspondence:

Yves Soyer

170 Avenue de la République - 91230 Montgeron

E-mail: yves.soyer@orange.fr

Article received: 12-10-2016. Accepted for publication: 02-12-2016.

This is an Open Access article distributed under the terms of the Creative Commons Attribution License (http://creativecommons.org/licenses/by/4.0), which permits unrestricted use, distribution, and reproduction in any medium, provided the original work is properly cited. 


\section{CLINICAL SEMIOLOGY}

Class-III malocclusions are anomalies where the mandible occupies a position that is significantly anterior in relation to the maxilla. These malocclusions comprise all cases of maxillary retrognathism and cases of mandibular prognathism.

If the skeletal and occlusal Class-III malocclusions are most often associated, Class-III occlusal relationships can be encountered on a Class-I skeletal pattern and conversely a dental Class I on a Class-III skeletal pattern.

Historically, Angle (1907) characterized Class III as "mesial occlusion of the lower jaw and arch, as indicated by the occlusion of the first mandibular molars at the time of their eruption."

This definition assigns responsibility for malocclusions to the lower jawbone and implies, on the one hand, the fixity of the upper jawbone as a skeletal and dental reference base and on the other hand, the consistency of the anomalies at the mandibular level, whether at the dental and/or mandibular body level.

Therefore, the terms Class-III malocclusion and mandibular prognathism were (and still are) often used interchangeably ${ }^{5}$.

Class-III malocclusions have many different clinical forms that influence their treatment.

\section{Class-III malocclusions related to malformative syndrome}

The cranial or facial involvement of different major syndromes lead to the development of a severe skeletal ClassIII malocclusions: Crouzon syndrome,
Binder syndrome, labioalveolar palatal clefts, achondroplasia, craniofacial stenosis, Down's syndrome, acromegaly, or Pierre Marie's syndrome. The prognosis of these cases does not allow for the consideration of orthopedic or interceptive treatments.

\section{Class-III malocclusions of kinetic origin}

Mandibular protrusion or "mandibular pseudoprognathism" or "mandibular anteposition" or "anti-mandibular" is functional in origin, without any anatomical bone deformation.

Clinical signs are the same as morphological prognathism. However, in the functional examination, a deviation from the posteroanterior closing path can be observed. This positional anomaly of the mandible is characterized by mandibular recoil and the restoration of the condyle at the bottom of the glenoid cavity, during the De Névrezé maneuver. This anterior position does not occur in skeletal Class-III malocclusions, but some structural Class-III malocclusions may be associated with sliding ${ }^{3}$.

\section{Structural Class-III malocclusions}

Delaire and Salagnac ${ }^{7}$ have individualized the different clinical varieties well.

\section{Maxillary Class-III malocclusions: "Maxillary insufficiency"}

Fifty percent of Class-III malocclusions are caused by maxillary insufficiency that results from one or more of the following five parameters: 


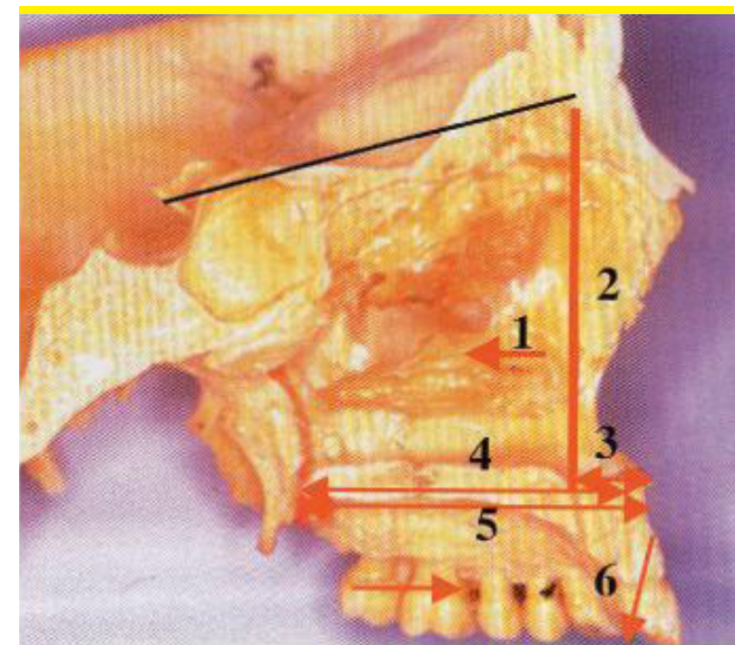

Figure 1

(C) Salagnac $J M^{21}$. The parameters

that can cause maxillary insufficiency.

- Maxillary orientation that is too posterior in relation to the reference to the cranial base: retrodisplaced maxilla (Fig. 1/1);

- Lack of maxillary height (Fig. 1/2);

- Lack of length of the palatal base: low brachymaxillia (Fig. 1/4) or prebrachymaxilla (Fig. 1/3);

The combination of these three parameters is a "micromaxillary deformity."

- removal of the alveolar arches: retroalveolia (Fig. $1 / 5$ );

- Dental: Abnormalities in the number of teeth: dental agenesis and poor dental position, in particular of the upper incisors (Fig. 1/6).

These different clinical entities require specific therapeutic protocols and

\section{ETIOPATHOGENESIS}

The prognosis and the treatment of Class-III malocclusions and the stability of results depend on the etiopathogenic diagnosis, which is all the more impor- many of these five parameters can, of course, exist together ${ }^{21}$ (Fig. 1).

\section{Mandibular Class-III malocclusions: "Mandibular excesses"}

The mandibular involvement may be global or concern only one or more regions. It is possible to differentiate the most frequent clinical entities: dolichoramia, dolichocorpia, macro-mandibulia, dolichocondyly, lower proalveolia, promandibulia, progeny, opening of the mandibular angle, with or without symphysial excess, and the mandible being too anterior in relation to the skull. Orthopedic or interceptive ${ }^{22}$ treatment has a bad prognosis in these last three varieties.

Jean Delaire summarizes his classification established in 1996 and was always authoritative in the interview he gave on mandibular prognathism syndrome ${ }^{6}$.

His multicenter study of 261 Class-III cases collected exclusively in private practice to avoid including too many "particular" subjects, on subjects aged 2.5-27 years and of which 45 were aged $<7$ years and 16 cases were aged $>13$ years, it was noted that the proportion of promandibulia increased considerably with age $140 \%$ in children aged $<7$ years and $87.50 \%$ in subjects aged $>14$ years).

It is, therefore, necessary to consider treating Class-III malocclusions as soon as possible.

tant as the clinical forms are varied. "The causes are too intertwined to be classified in a strictly chronological or primordial order."14 


\section{Hereditary Causes}

Skeletal Class-III disorders, and especially cases of mandibular prognathism, are dysmorphisms whose hereditary character has been widely demonstrated.

Victor Galippe in 1905, Oswald Rubbrecht $^{20}$ in 1929, and Jacobson ${ }^{12}$ in 1974, have made remarkable studies on the mandibular prognathism of the Habsburgs where, out of 40 members, there were 33 cases of prognathism.

In the course of their clinical examination, the practitioner will focus on finding the malformation in the patient's family, which will allow them to ascertain the prognosis. In fact, this finding alone can help in assessing the prognosis.

\section{Hormonal Causes}

Hyperfunction of the pituitary gland may cause gigantism during growth and eosinophilic adenoma of the sella turcica can lead to acromegaly in adults ${ }^{16}$.

\section{Muscular and functional environ- ment Labiolingual muscular envi- ronment}

The tongue plays a very important role in the development of Class-III malocclusions because of its rather increased volume, its low and anterior position, its tonicity and its functional behavior. Indeed, when swallowing and speaking, the dysfunctional lingual behavior will have a double morphogenetic consequence.

On the lip level, the inverted interincisal ratio leads to labiomentonary hyperactivity at rest, especially marked at the lower orbicular level in mandibular prognathism ${ }^{3}$.

\section{Dysfunctional environment}

Mouth breathing plays a major etiological role in Class-III cases by imposing a low and often anterior tongue position in cases of associated tonsillar hypertrophy.

With regard to the other functions such as chewing, swallowing, phonation, one must be well aware that there is a veritable "vicious spiral of malfunctions" which originates from mouth breathing causing abnormalities of posture and altering all the functions of the cephalic extremity. This favors facial sagittal development in Class-III patients ${ }^{26}$.

prevent the emergence of a dysmorphism.

Interception treatments occur when the malocclusion is already present, at a more or less advanced stage, while prevention occurs before the dysmorphism occurs ${ }^{23}$. 
In the face of Class-III dysmorphisms, there is some consensus as to when to implement a treatment that corrects the pathology.

The interceptive therapy of Class-III malocclusions is part of a growth pattern and will be introduced as soon as possible in simple cases.

The therapeutic intervention is therefore precocious to restore the incisor recovery as soon as possible and to standardize the functions so as to steer and normalize the subsequent growth.

The aim is to restore, as soon as possible, the form and function so as to re-establish good maxillofacial relations and a good orientation of the maxilla in relation to the cranial base by allowing a good balance and development of nasal ventilation ${ }^{13}$.

\section{Correction of mandibular shifting}

Its correction is indispensable to avoid the evolution of skeletal ClassIII malocclusions. It will be appropriate to remove any occlusal interference by grinding the cusps of temporary canines. A transverse expansion can be associated with a Class-III activator.

\section{Correction of neuromuscular behavior}

From an early age, it is appropriate to re-establish normal orofacial functional behaviors so as to normalize the position (low and anterior) and lingual functions by performing a lingual frenectomy with kinesthetic re-education, by reestablishing nasal ventilation and freeing the airways with an adenoidectomy and tonsillectomy if necessary.
The use of a nocturnal lingual envelope or functional Class-III plate will promote lingual repositioning and incisor overlap.

Of course, any parafunction (onychophagy, behavioral attitude, and sucking) favoring the establishment of a ClassIII malocclusion will be eliminated.

\section{Orthopedic Therapy}

The therapeutic objective is to stimulate the growth of the upper maxilla and to redirect or inhibit the growth of the mandible.

\section{Occipital Chin Sling}

It is used with very young children in cases of mandibular prognathism with no apparent maxillary deficiency. This device will promote the closure of the gonial angle and the slowing of mandibular growth. It is up to the parents to insist on its importance because it is them who, doubting its effectiveness, do not always promote wearing it seriously.

\section{Anterior-Posterior Extraoral Forces}

They can be applied to the mandible: braces are placed on permanent molars or on temporary second molars. Chabre and $\mathrm{Canal}^{4}$ have thus demonstrated the decline of the lower molar on its base and the correction of the molar Class-III ratios, as well as a tendency to correct Class-III ratios of the bases (of course, they remain contraindicated in terms of vertical growth.)

\section{Posterior-Anterior tractions on the maxilla: Delaire face mask}

Developed by Delaire and Verdon, it is a simple method that has revolutionized the treatment of Class-III 
malocclusions caused by maxillary insufficiency. The period of temporary teeth, i.e., age 4-12 years, remains the most favorable period.

The harmony of bone bases is improved rapidly as well as incisor recovery to enable the restoration of a more balanced growth.

With the support of the frontal and chin regions, the mask exerts important tractions on the upper dental arch.

From an intraoral anchorage (double rigid arch sealed on the upper molars), posteroanterior tractions are exerted through elastics.

These pulls can be increased gradually from $200 \mathrm{~g}$ for a young child. Depending on the age of the patient, the importance of the desired component and the speed of the desired correction, it is possible to exercise traction of $1500 \mathrm{~g}$.

Verdon and Salganac ${ }^{21}$ have observed exceptional opportunities for action:

- Maxillary:

- A change in the maxillary orientation in relation to the cranial base;

- an increase in the length of the maxillary base ([NA-[NP);

- An increase in the distal distance of the last molar to the maxillary tuberosity.

- Mandibular:

- A slight lowering and retraction of the mandible with sometimes increase anterior facial height: [NA-Chin.]

- Dental:

- a constantly favorable change in the orientation of the upper incisors in relation to the premaxillary;

- Improved dental relationships and the orientation of the occlusion plan.
- Profile:

- Improved facial esthetics through improved interlabial relationships;

- A progressive filling of the nasolabial hollows and the sub-orbital areas.

The treatment is in fact a compensatory treatment: while stopping the growth of the mandible, one advances orthopedically the maxilla, taking care to physiologically orient the occlusion plan $^{19}$.

\section{Activators}

These intraoral device modify interarch relationships to decrease skeletal lag and normalize occlusal relationships, especially at the anterior level to harmonize subsequent maxillary and mandibular growth.

Construction wax is applied while the mandible is in the most retrusive position and it increases the vertical dimension of the mandible.

The activator consists of a resin monobloc with an important lateral resin interposition to deprogram the temporomandibular joints and allow progressive grinding from rear to front to move the mandible backward to a position of comfort respecting the minimum vertical dimension of occlusion. Occlusal elevation contributes to distal mandibular repositioning.

An Eschler arc is positioned to maintain the mandible in the most retrusive position and to maintain the orientation of the lower incisors by avoiding a linguoversion.

By muscular play, the mandible tends to move forward previously repulsing the activator, which thus transmits a posteroanterior and transverse stimulation to the maxilla. 
The Class-III activator thus makes it possible to observe ${ }^{13}$ :

- Muscular deprogramming;

- A correction of the forward shift;

- A centered adjustment;

- Stimulation of maxillary growth and a mesial slippage of the maxillary arch;

- A slowing of mandibular growth and a distal slippage of the mandibular arch;

- Closure of the gonial angle;

- Verticalization of the growth direction.

\section{Class-III intermaxillary traction on microplates}

De Clerck's bone-anchored maxillary protraction (BAMP) protocol, developed in the growth phase, proposes the use of Class-III intermaxillary traction on microplates with temporary anchors, allowing orthopedic maxillary protraction to avoid the dentoalveolar extraneous effects when the traction devices are applied to the dental arches.

This new paradigm allows the treatment of borderline cases by interception therapy, rather than delaying treatment and waiting for the end of the growth stage to undergo orthodontic surgery ${ }^{11}$ (Fig. 2).

This protocol, which appears to be a major development for the interception of Class-III cases, has been associated with a pterygomaxillary disjunction with the aim of:

- potentiate the progress of the maxillozygomatic complex in one unit;

- Decrease the disparities on the maxillary protraction obtained with the BAMP protocol;

- Obtain results in older patients with CS4 and CS5 cervical maturation.

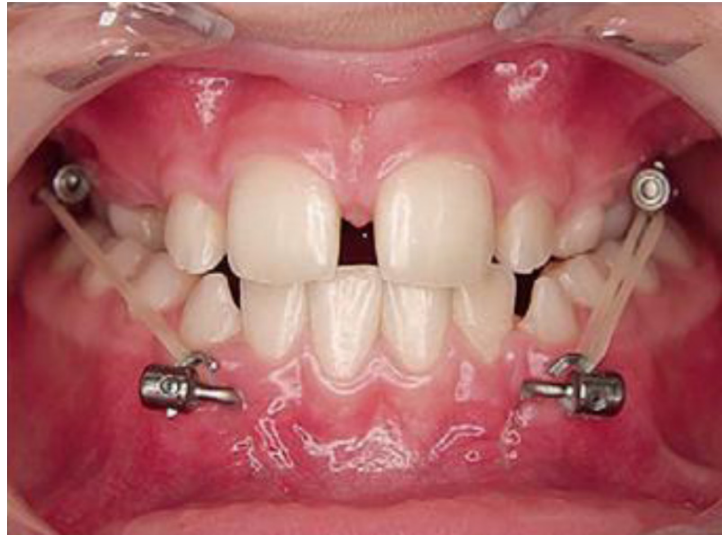

Figure 2

(C) De Clerck $H^{11}$.

The weakening of the pterygomaxillary suture would increase the performance of the zygomatic maxillary suture with reciprocal action. The advance in mass of the zygomatic maxillary complex would be similar to that obtained with LeFort III surgery.

This is all the more interesting as patients with Class-III dysmorphisms present for the most part, aplasia of the entire skull base ${ }^{9,10}$.

\section{Interceptive Orthodontic Treatment}

Interceptive therapy can act directly on dental arches.

The aim is to restore a maximal intercuspidal occlusion centered without muscular restraint in the context of dental Class-I occlusion allowing an alternating unilateral chewing ${ }^{18}$.

For the chewing to physiologically practical, the occlusal plane must be oriented to a line approximately parallel to the plane of Camper $^{15}$ (Fig. 3). 


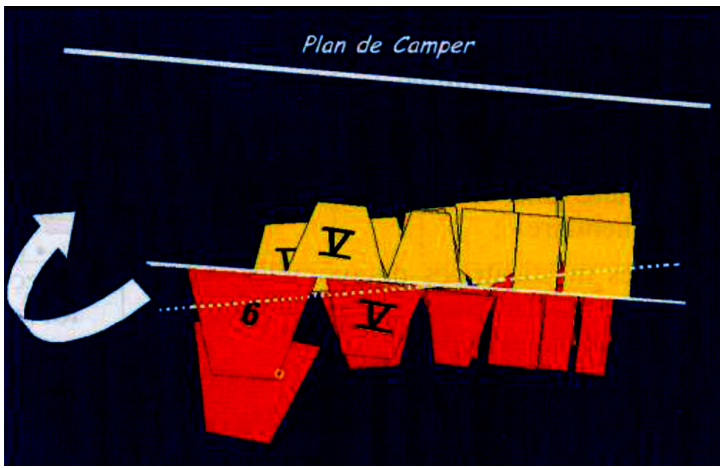

Figure 3

(C) Raymond $J L^{17}$. Diagram of rotational direction of the occlusal plane. Dotted white line: initial pathological orientation, down and backward. Full white line: final orientation approximately parallel to the Camper plane (for didactic reasons the divergences are exaggerated).

It is therefore necessary to seek the harmony between the determinants of the occlusion, described by Hanau, according to the formula:

Equilibrium $=$ (incisor slope $\times$ condylar slope) / (cuspidal height $\times$ inclination of the occlusion plane $\times$ curve of SPEE).

Early occlusal and functional standardization will:

- stop the evolution of the dysmorphism by breaking the "dysmorphofunctional" spiral;

- the potentiation of the correction thanks to the rehabilitated physiolog-

\section{INTERCEPTION, IS IT AN OBLIGATION?}

Do clinicians have to "shoot everything that moves" and intervene as soon as the slightest sign of dysmorphism manifests itself, even early in childhood ${ }^{8}$ ?

Each patient will respond according to their sensitivity: prevention from

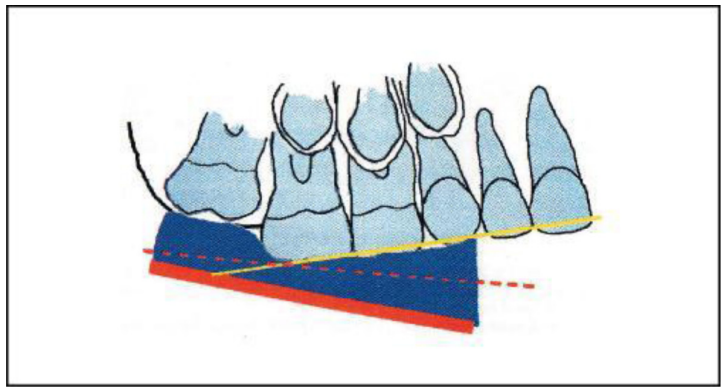

Figure 4

(c) Raymond $\mathrm{JL}^{17}$. The thickness of the plate (in blue) is the maneuverable space used for the reorientation of the occlusal plane. In yellow, the initial pathological orientation of the occlusal plane. In red dots, the desired orientation at the end of treatment and in red (solid lines), the initial orientation of the plate. (Note: The inclinations drawn are exaggerated to make the diagram more didactic.)

ical mastication, which can be an ally of the practitioner;

- stability of the therapeutic result because the early reorientation of the occlusion plane creates the conditions for a "peaceful" eruption of the posterior teeth, i.e., without disrupting the mandibular kinematics ${ }^{17}$.

The treatment procedure is performed by the interposition of a plate, which will be selectively grinded at the occlusal side according to the orientation of the desired occlusal plane (Fig. 4).

temporary dentition, interception during stable mixed dentition, orthodontics in adult permanent teeth or inevitable surgery.

Invented in Canada in the 1980 years, evidence-based medicine was 
introduced into our specialty in the 2000s and was able to sow doubt and mistrust in clinicians' minds on the pretext that their interceptive practice could not be based on any randomized study.

The High Authority of Health an independent public authority, contributes to the regulation of the health system and recommends:

"For specific cases of Class-III occlusions, (...) to treat mandibular forward shifts as soon as possible (...) The treatment of Class-III functional malocclusions must be implemented as early as possible (rehabilitation of orofacial and maxillary advanced functions using the Delaire mask)"
In addition, the American Academy of Pediatric Dentistry advocates the interception of Class-III malocclusions to provide psychosocial benefits to the child by decreasing or suppressing facial disfiguration and decreasing the severity of the malocclusion by promoting harmonious growth?.

When faced with a Class-III malocclusion, clinicians can no longer say that it is better to wait.

Ignoring interceptive therapy leads to the professional responsibility of the practitioner for failure to provide information on the various therapeutic possibilities and for missed opportunity because he has caused his patient to lose the choice of early orthodontic treatment ${ }^{2}$.

\section{CONCLUSION}

Henriette Muller's article on the "skeletal Class-III" probably has given rise to research, studies, and publications.

Class-III malocclusions can affect all skeletal, alveolar, and dental structures. Because the organism adapts to dysmorphism, the clinical forms are generally mixed and the constituents involved (cranial typology, maxillary size, and their joint relationships with the cranial base) can be amplified or compensated.

In the face of Class-III dysmorphisms, the interceptive approach performed during the growth period must prevent the onset of dysmorphisms and prevent their aggravation to facilitate a normal growth direction of the bone bases.

According to Planas, on the principle of Claude Bernard: "The function creates the organ and the organ adapts to the function."

"Primum non-nocere" is a medical notion that must guide our therapeutic decisions. The "growth" factor and the "time" factor are essential elements in the development of Class-III disorders.

Conflict of Interest: The author states that he has no conflict of interest.

\section{BIBLIOGRAPHY}

1. Amat P.Traitement précoces des malocclusions de classe III: les faits. Orthod Fr 2013;84:41-52.

2. Béry A. Le préjudice réparable: la perte de chance. Orthod Fr 2013;84:15-27. 
3. Boileau MJ, Duhart AM. Classes III, sémiologie et principes thérapeutiques. EMC 2014;9(4):1-12 [article 23-472-G-05].

4. Chabre C, Canal P. Étude de l'action dentaire et sque-lettique des forces extra-buccales antéro-postérieures appliquées à la mandibule. Orthod Fr 1979;50:433-446.

5. Delaire J. Cours ARREMAC, Nantes 2012.

6. Delaire J, De Brondeau. À propos du syndrome prognatique mandibulaire. Rev Orthop Dento Faciale 2017;51.

7. Delaire J, Salagnac JM, Notari J. Diagnostic des dysmorphoses dento-maxillo-faciales. Apport de l'analyse architecturale informatisée. Actual Odontostomalol 1994;75(7):951-970.

8. Faure J. Traitements très précoces, précoces, bien expectative armée? Orthod Fr 2013; 84:71-85.

9. Ghnassia M, Racy E. Interception des classes III squelettiques par tractions intermaxillaires sur ancrages osseux: Intérêts de la disjonction ptérygo-maxillaire associée. Communication affichée. FFO Les 19eJournées de I'Orthodontie, Paris, 2016.

10. Ghnassia M, Racy E. Interception des classes III squelettiques par tractions intermaxillaires sur ancrages osseux assistée par chirurgie mini-invasive. FFO Les 19eJournées de I'Orthodontie, Paris, 2016.

11. Heymann GC, Cevidanes L, Cornelis M, De Clerck H, Tullock JFC. Three dimentional analysis of maxillary protraction with inter-maxillairy elastics to miniplates. Am J Orthod Dentofac Orthop 2010;137:274-284.

12. Jacobson A, Evans WG. Mandibular prognatism. Am J Orthod 1974;66;2:140-171.

13. Le Gall M, Philip C, Salvadori A. Traitement précoce des classes III. Orthod Fr 2011;82:241252.

14. Muller H. Classe squelettique III. Rev Orthop Dento Faciale 1970;I:17-49.

15. Planas P. La réhabilitation neuro-occlusale. Paris: Masson, 1992.

16. Perrier d'Arc G. Prognathie mandibulaire. Encycl Méd Chir, Paris, Stomatologie 23472 G-10, 4-1979.

17. Raymond JL. Finalité fonctionnelle et occlusale du traitement orthopédique de classe III. Rev Orthop Dento Faciale 2003;37:285-303.

18. Raymond JL. Justification occlusale du traitement précoce de la classe III. Orthod Fr.

19. Raymond JL. Traitement orthopédique des classes III. Réhabilitation occlusale et fonctionnelle. St Laurent: Empresa, 2011.

20. Rubbrecht O. Type familial des Habsbourg. Revue de Stomatologie 1929;2.

21. Salagnac JM. Traitements des classes III. Orthod Fr 2006;77:187-206.

22. Salagnac JM. Développement normal et pathologique de la mandibule. Déductions pratiques en orthopédie maxillo-dento-faciale. Orthod Fr 2016;87:293.

23. Salvadori A. Interception. Rev Orthop Dento Faciale 1987;21:185.

24. Salvadori A, Martin-Lacombe M. Orthodontie fonctionnelle. Encycl Med Chir, Paris, Stomatologie II 23494A10 5-1983.

25. Soyer Y. Interception des malocclusions de classe III d'Angle. Rev Orthop Dento Faciale 1987;21:235-249.

26. Vesse M. Classes III squelettiques. Encycl Méd Chir, Paris, Odontologie/Stomatologie, 23-472-G-10, 1999, 18 p. 